\title{
Peripheral neuropathy complicating pancreatitis and major pancreatic surgery
}

\author{
M L P GROSS, CLARE J FOWLER, R HO, R C G RUSSELL, M J G HARRISON \\ From the Reta Lila Weston Institute of Neurological Studies and the Department of Surgery, The Middlesex \\ Hospital Medical School, London, UK
}

SUMMARY Four cases of a polyneuropathy associated with pancreatitis and pancreatic pseudocyst formation are reported. Electrophysiological investigation showed the peripheral neuropathy to be predominantly axonal in type. These patients were all seriously ill and many factors may have been involved in the pathogenesis of their neuropathy. They had all received parenteral nutrition and multiple drug therapy including metronidazole, and all had severe sepsis. There was evidence that insufficient vitamin replacement had been given during total parenteral nutrition. It was not possible to decide whether the polyneuropathy resulted from the summation of these factors, is similar to what has been called the polyneuropathy of the critically ill, or is a new association with pancreatic disease.

Increasing surgical skill and the use of prolonged parenteral nutrition have enabled patients with severe pancreatic disease to be managed successfully. Unfortunately in the four cases reported here, a polyneuropathy developed during the course of the illness and its convalescent phase and delayed the patients' ultimate recovery. A neuropathy of the critically ill has recently been described ${ }^{12}$ but the cause remains uncertain. It does not seem to be a form of GuillainBarré polyneuropathy although it is known that this may complicate surgery or serious illnesses. ${ }^{3}$ A particular association of polyneuropathy with pancreatic disease has not been reported and it is not certain if our cases represent a new association or form part of the spectrum of cases with polyneuropathy of critical illness. In the four cases reported here, we have examined the role of parenteral nutrition, drug therapy and sepsis in the pathogenesis of the neuropathy.

Address for reprint requests: Dr M Gross, The National Hospital for Nervous Diseases, Queen Square, London WCIN 3BG, UK.

Received 15 January 1988 and in revised form 19 May 1988 Accepted 23 May 1988

\section{Case reports}

Case 1

A 17 year old woman was found to have hyperparathyroidism and a renal calculus; a parathyroid adenoma was excised in May 1985. A postoperative pyrexia was treated with a course of metronidazole, amoxycillin and flucloxacillin in standard dosage. Within $\mathbf{4 8}$ hours of surgery she developed severe abdominal pain. The serum amylase was elevated and ultrasound of the abdomen demonstrated acute pancreatitis with a pancreatic pseudocyst. Total parenteral nutrition was started, with percutaneous drainage of the pseudocyst with the insertion of a catheter under ultrasound control. On the 12 June 1985 she was restarted on broad spectrum antibiotics together with metronidazole and this treatment was continued for 2 months. In early August 1985 , after 3 months of total parenteral nutrition, and at a time when the antibiotics had stopped, she developed pains in her legs with pins and needles and marked weakness.

On examination there was minimal distal wasting of the small muscles of her hands and feet. She had global weakness, more marked distally than proximally. The triceps, knee and ankle reflexes were absent, the biceps and supinator reflexes were present and both plantar responses were flexor. Tactile sense and pain sensation was impaired over the fingers and toes. Vibration sense was absent at the ankle but postural sense was preserved. Electrophysiological investigations confirmed the presence of an axonal neuropathy with evidence of denervation in the small distal muscles sampled. No cause of the peripheral neuropathy was 
discovered on investigation over many months. She made a very slow spontaneous recovery, and when reviewed in October 1985, she was walking, and her reflexes had returned. She was able to work fulltime.

\section{Case 2}

A 53 year old man developed acute pancreatitis in August 1985. There was no previous history of alcohol abuse or biliary disease. After medical management he had a further attack in November 1985 and developed nodules on both legs, a biopsy specimen of which showed subcutaneous fat necrosis. A diagnosis of acute pancreatitis with pseudocyst formation secondary to pancreas divisum was made and from November 1985 he received parenteral nutrition. On the 20 December 1985 he underwent distal pancreatectomy, splenectomy, pancreato-jejunostomy and cholecystectomy. The postoperative course was complicated with transient episodes of insulin provoked hypoglycaemia which were rapidly controlled. On 5 January 1986 he was noted to have weakness in his legs and some limb pain. Examination revealed wasting of the proximal leg muscles with marked reduction in power (MRC Grade I). He was areflexic in the legs with preservation of the arm reflexes. Both plantar responses were flexor. There was a mild distal loss of vibration sensation but the other sensory modalities were normal. Electrophysiological investigations confirmed the clinical diagnosis of a predominant motor neuropathy with motor conduction velocities at the lower limit of normal and delayed or absent $F$ waves. He continued to make a slow recovery from his neuropathic process, regaining normal power in the legs by January 1987.

\section{Case 3}

A 31 year old male developed severe abdominal pain on 8 November 1985. At laparotomy he was found to have acute pancreatitis. By 19 November 1985 he had developed subhepatic and subphrenic abscesses together with a pancreatic pseudocyst and was started on total parenteral nutrition. From 21 November he was started on metronidazole which continued for 2 months together with other antibiotics. On 18 January 1986, he underwent a pancreato-jejunostomy. By 6 February 1986 it was noted that he was having difficulty walking with weakness in his limbs and distal sensory disturbance in both hands and feet. There was no pain. On examination there was weakness of muscles supplied by the 5 th and 7 th cranial nerves. There was marked wasting of the small muscles of the hands and feet and tibialis anterior bilaterally. There was global limb weakness but this was most marked distally in the legs, there being no movement below the knee. He had absent triceps, knee and ankle reflexes but both biceps and supinator jerks were present. There were no plantar responses. He had a glove and stocking sensory loss to all modalities. Light touch was lost to the knee, pin-point sensation was lost to the ankle and vibration sense to the knee. Electrophysiological investigation confirmed the severe generalised neuropathy which was predominantly axonal in type with evidence of denervation in the small hand muscles. Extensive investigations revealed no other abnormality to account for the neuropathy. The vitamin levels were normal except for vitamin $\mathrm{B} 1$. The red cell basal transketolase activity was 0.41 $\mathrm{mmol} / \mathrm{g}$ of haemoglobin (normal values $0 \cdot 42-1 \cdot 22 \mathrm{mmol} / \mathrm{g}$ ).
The vitamin effect was abnormal at $25.7 \%$ (normal values $0-20 \%$ ) with an activity of 1.39 (normal: $1.0-1 \cdot 2 \mathrm{mmol} / \mathrm{g}$ ). Trace element analysis for zinc, copper and magnesium were also normal. At the present time he is slowly improving but has developed pain in his limbs.

\section{Case 4}

A 36 year old man complained of abdominal pain and vomiting on 8 September 1982 . He had recently lost $6 \mathrm{~kg}$ in weight. He subsequently developed an obstructive jaundice. Ultrasound examination demonstrated a mass in the head of the pancreas. At laparotomy on 28 October, a pseudocyst was found together with a disordered pancreatic duct system and the pseudocyst was drained. Total parenteral nutrition had been introduced over this period. On 23 November 1982 he became confused, toxic, sweaty and ill with septicaemia and treatment with gentamicin, amoxycillin and metronidazole was commenced. Over the next 48 hours he became increasingly drowsy and confused. He moved his legs less well. On examination he was disorientated, responding only to simple commands. He had bilateral 6th nerve palsies with horizontal nystagmus. In the limbs, there was bilateral foot drop with weak and wasted proximal leg muscles and absent reflexes. Only the right biceps and both supinator reflexes were obtainable. The plantar responses were flexor. Pinpoint sensation was impaired below the knees. Fine joint position sense was absent in the toes as was vibration sense. Nerve conduction studies showed a predominantly axonal sensorimotor polyneuropathy. Treatment of the sepsis combined with vitamin therapy led to the resolution of the confusional state and 6th nerve palsy. Initially there was mild confabulation and poor memory though this improved with continued parenterovite therapy. The neuropathy slowly improved over many months though a mild disturbance of gait was still apparent in December 1986. The patient finally underwent a radical distal pancreatectomy and cholecystectomy on 30 March 1983 . He received total parenteral nutrition and intensive vitamin therapy and no further problems were encountered.

\section{Total parenteral nutrition}

All four patients received total parenteral nutrition. A standard regime was used. ${ }^{4}$ The patients received 2.5 litres of fluid in a single bag containing 2,400 calories of which $20 \%$ was lipid, $350 \mathrm{~g}$ carbohydrate and $14 \mathrm{~g}$ on nitrogen. Each bag contained $73 \mathrm{mmol}$ of sodium, $60 \mathrm{mmol}$ potassium, $30 \mathrm{mmol}$ phosphate, $5 \mathrm{mmol}$ magnesium and $70 \mathrm{mmol}$ chloride. Once every four days, one $10 \mathrm{ml}$ vial of Solivito (KabiVitrum-

Table Vitamin replacement during total parenteral nutrition

\begin{tabular}{lcc}
\hline & $\begin{array}{l}\text { Solivito } \\
(\mathrm{mg})\end{array}$ & $\begin{array}{l}\text { Recommended daily minimum } \\
\text { (Truswell 1985 } \mu \mathrm{g})\end{array}$ \\
\hline Vitamin B1 & $1 \cdot 2$ & $1 \cdot 0$ \\
Vitamin B2 & $1 \cdot 8$ & $1 \cdot 5$ \\
Vitamin B6 & $2 \cdot 0$ & $3 \cdot 0$ \\
Nocotinamide & $10 \cdot 0$ & $18 \cdot 0$ \\
Pantothenic acid & $10 \cdot 0$ & $5 \cdot 0$ \\
Biotin & $0 \cdot 3$ & $0 \cdot 1$ \\
Folic acid & $0 \cdot 2$ & $0 \cdot 2$ \\
Vitamin C & $30 \cdot 0$ & $30 \cdot 0$ \\
\hline
\end{tabular}


table) and Vitalipid (KabiVitrum) was added to the bag and three times a week Adamel (KabiVitrum) $10 \mathrm{ml}$ was added. All four patients received folic acid $15 \mathrm{mg}$ once daily.

\section{Discussion}

Four cases of an acute or subacute axonal neuropathy are reported complicating pancreatitis and pseudocyst formation. In three cases, major pancreatic resection was undertaken and in the fourth case, repeated pseudocyst drainage. These patients have a number of features in common but it is not clear which are involved in the pathogenesis of their peripheral neuropathy. A polyneuropathy complicating critical illness has been described in patients with no evidence of heavy metal poisoning, porphyria, viral infection or specific vitamin deficiency. ${ }^{12}$ Many of these patients had received antibiotics of the aminoglycoside group and other combinations including metronidazole. All the patients suffered sepsis at the time and the possibility of neuropathic bacterial toxins could not be excluded. Nutritional deficiency was considered in these patients as the depressed levels of blood albumin improved after the institution of total parenteral nutrition. The polyneuropathy of the critically ill can be differentiated from the Guillain-Barre syndrome by the electrophysiological features and the normal levels of protein in the cerebrospinal fluid.

We are not aware of any previous reports of a peripheral neuropathy associated with pancreatitis or pancreatic surgery. A distal sensory axonopathy can complicate insulinomas and there are 30 cases in the world literature. ${ }^{5}$ However, only one of our patients was hypoglycaemic and that too briefly to make this a likely explanation for the neuropathy. Patients with acute pancreatitis can develop a "pancreatic encephalopathy" which has been thought due to nicotinic acid deficiency ${ }^{6}$ though this hypothesis has been doubted by others. ${ }^{7}$ Vogel $^{8-10}$ injected hog pancreatic lipase into rabbit brain and produced focal areas of demyelination and similarly bathing spinal cord segments in lipase produced demyelination whereas injection of trypsin or chymotripsin produced only tissue necrosis. Whether lipase has similar demyelinating effects in the peripheral nervous system does not seem to have been studied. Pancreatic diarrhoea produced by APUD series neuropeptide secreting tumours can induce generalised weakness but this is due to potassium loss and secondary muscle weakness rather than a neuropathic process. ${ }^{11}$ In any case, none of our patients was hypokalaemic.

All four cases received treatment with metronidazole. Ursing \& $\mathrm{Kamme}^{12}$ reported a sensory neuropathy associated with the use of metronidazole in Crohn's disease and further cases have been reported $^{13}$ in whom a sensory peripheral neuropathy was associated with prolonged oral metronidazole use. The motor neuropathies in our patients seemed to be quite different from the sensory neuropathies with metronidazole and in two the neuropathy developed after the metronidazole was stopped. The possibility that the combination of metronidazole therapy and vitamin deficiency has a more potent neuropathic effect remains. We considered the possibility of a postoperative Guillain-Barré syndrome (acute inflammatory polyneuropathy). The CSF was not examined in the acute phase of the neuropathy in these patients. An elevation in the CSF protein concentration would have supported the diagnosis of Guillain-Barré syndrome as was seen in the eight cases of postoperative Guillain-Barré syndrome reported by Arnason and Asbury. ${ }^{15}$ The nerve conduction studies in all four cases would have been atypical in that there was evidence of predominant axonal degeneration, and clinically there was striking preservation of some upper limb reflexes. This diagnosis however remains a possibility for Case 3 especially in view of the absent $F$ waves and associated facial diplegia.

All the patients received extended total parenteral nutrition over many months and the possibility remains that in some way this could be related to the neuropathic process. A standard regime of total parenteral nutrition was followed similar to that recommended in the British National Formulary. ${ }^{4}$ None of our patients developed diabetes with their total parenteral nutrition and pancreatitis although in two cases intravenous insulin was used in the postoperative period which led to intermittent hypoglycaemia in one case.

The table demonstrates the quantity of vitamin contained in Solivito (KabiVitrum) compared with the daily recommended minimum. ${ }^{16}$ All four cases received considerably less vitamin replacement than that recommended as daily requirement and this assessment does not take into account the extra vitamin usage associated with sepsis, the large carbohydrate loads of parenteral nutrition and the possible degradation of the vitamin solution that then takes place. It is unlikely that thiamine deficiency alone was responsible for the neuropathy. The rapidity of onset after a relatively short period of vitamin depletion would be surprising. Also the neuropathies associated with vitamin B group deficiency tend to be sensory and painful, with the exception of that related to vitamin B12. The dominant non-painful motor involvement in three cases is thus a pointer against $B$ vitamin deficiency as a primary cause. The amount of Vitalipid added was also less than the daily recommended minimum. There is no vitamin $\mathrm{E}$ in Vitalipid and that contained in Intralipid (soya oil) has only $10 \%$ activity. Unfortunately, vitamin E levels were not measured and we do not know if a deficiency state 
prevailed. It is probable that many patients with critical illness become vitamin depleted, particularly if vitamin replacement is not given from the onset of their hospital admission. We recommend that vitamin therapy should be greater than that regarded as the daily minimum required in normal people. In our patients this would have meant giving at least two ampoules of Solivito daily rather than the twice weekly regime that is recommended. ${ }^{4}$ It seems possible that the combination of pancreatic disease, vitamin deficiency, sepsis, metronidazole therapy and surgery together with short term glucose intolerance may have a summation effect. Alternatively, this may represent a new, previously unreported complication of severe pancreatic disease.

\section{References}

1 Bolton CF, Laverty DA, Brown JO, Witt NJ, Hahn AF, Sibbald WJ. Critically ill polyneuropathy: electrophysiological studies and differentiation from Guillain-Barré syndrome. $J$ Neurol Neurosurg Psychiatry 1986;49:563-73.

2 Zochodne DW, Bolton CF, Wells GA, Gilbert JJ, Hahn AF, Brown JD, Sibbald WJ. Critical illness polyneuropathy. Brain 1987;110:819-42.

3 Gross MLP. The Therapeutic Modification of Inflammatory Polyneuropathy. MD thesis, University of Cambridge 1985.

4 British National Formulary (1987) Chapter 9, p. 298.
British Medical Association. The Pharmaceutical Press.

5 Jaspan JB, Wollman RL, Bernstein L. Hypoglycaemic peripheral neuropathy in association with insulinomas: implication of glycosopaenia rather than hyperinsulism. Case report and literature review. Medicine 1982;61:33-44.

6 Rothermich NO, Von Haam E. Pancreatic encephalopathy. J Clin Endocrinol \& Metab I 1941:872-81.

7 Pallis CA, Lewis PD. The Neurology of Gastrointestinal Disease. WB Saunders-Philadelphia. 1984.

8 Vogel FS. Demyelinisation induced experimentally by means of lipase. (Abstract) Fed Pro 1951;9:347.

9 Vogel FS. Demyelinisation induced in living rabbits by means of a lipolytic enzyme preparation. $J$ Exp Med 1951;93:297-304.

10 Vogel FS. Cerebral demyelination and focal visceral lesions in a case of acute haemorrhagic pancreatitis with a consideration of the possible role of circulating enzymes in the causation of lesions. Arch Pathol, 1952;52:255-362.

11 Layzer RB. Neuromuscular Manifestation of Systemic Disease. Contemporary Neurology Series Vol. 25. Philadelphia: F A Davies, 1985.

12 Ursing B, Kamme C. Metronidazole for Crohn's disease. Lancet 1979;i:775-7.

13 Coxon A, Pallis CA. Metronidazole neuropathy. $J$ of Neurol, Neurosurg Psychiatry 1976;39:403-5.

14 Duffy LF, Damm F, Fisher SE, et al. Peripheral neuropathy in Crohn's disease patients treated with metronidazole. Gastroenterology 1985;88:681-4.

15 Arnason BG, Ashbury AK. Idiopathic polyneuritis after surgery. Arch Neurol 1986;18:500.

16 Truswell AS. Vitamins. I. Br Med J 1985;291:1003-5. 